\title{
SELETIVIDADE DE INSETICIDAS SOBRE O COMPLEXO DE PREDADORES DAS PRAGAS DO ALGODOEIRO ${ }^{1}$
}

\author{
Izidro dos Santos de Lima Júnior ${ }^{2}$, Rodrigo Fernandes Nogueira², \\ Thiago Ferreira Bertoncello², Elmo Pontes de $\mathrm{Melo}^{2}$, Renato Suekane², Paulo Eduardo Degrande
}

\begin{abstract}
SELECTIVITY OF PESTICIDES OVER

PREDATORS OF COTTON PLANT PESTS

The cotton plant hosts a complex of pests that can damage plant structures. Its supported development, in this agroecosystem, demands the implementation of an integrated pest management (IPM) system. The goal of this research was to study the selectivity of pesticides over predators of cotton plant pests. The experimental design was randomized blocks, with 9 treatments ( 84 days after the emergence) and 4 replicates. The sampling involved the beat cloth method, with 5 beats per plot, allowing to identify and count the living predators. Clotianidin 500 WP (200 $\left.\mathrm{g} \mathrm{ha}^{-1}\right)$, Carbosulfan $400 \mathrm{SC}\left(400 \mathrm{~mL} \mathrm{ha}^{-1}\right)$, Benfuracarb $400 \mathrm{EC}\left(450 \mathrm{~mL} \mathrm{ha}^{-1}\right)$, Cartap hydrochloride 500 SP $\left(1,000 \mathrm{~g} \mathrm{ha}^{-1}\right)$, Thiamethoxam $250 \mathrm{WG}\left(200 \mathrm{~g} \mathrm{ha}^{-1}\right)$, and Acetamiprid $200 \mathrm{SP}$ (150 $\left.\mathrm{g} \mathrm{ha}^{-1}\right)$ were not selective for the complex of the predators identified, with mortality percentages ranging from moderately toxic to toxic. Etofenprox $300 \mathrm{EC}$ $\left(450 \mathrm{~mL} \mathrm{ha}^{-1}\right.$ ) was the most toxic pesticide, when compared to the others treatments. The Flonicamid $500 \mathrm{WG}\left(150 \mathrm{~g} \mathrm{ha}^{-1}\right)$ treatment was selective, with average of predators similar to the control, until 8 days after spraying. Among the pesticides evaluated, only Flonicamid 500 WG was considered selective, according to the IOBC classification, because it showed the lowest mortality percentage for the complex of predators identified.
\end{abstract}

KEY-WORDS: Gossypium hirsutum L.; insecta; pesticides; cotton plant; predators.

\section{INTRODUÇÃO}

O algodoeiro é hospedeiro de um complexo de pragas, que podem ocasionar danos às estruturas das plantas. Os níveis populacionais destas pragas sofrem grandes variações, e infestações elevadas provocam sérios prejuízos à cultura, se o efetivo controle não for feito. As medidas de controle das pragas fazem parte dos sistemas de produção do

\section{RESUMO}

O algodoeiro é hospedeiro de um complexo de pragas, que podem ocasionar danos às estruturas das plantas. Para o desenvolvimento sustentado, neste agroecossistema, há necessidade da implementação do Manejo Integrado das Pragas (MIP). O objetivo deste trabalho foi estudar a seletividade de inseticidas aos predadores das pragas do algodoeiro. $\mathrm{O}$ delineamento experimental utilizado foi o de blocos ao acaso, com nove tratamentos e quatro repetições. Os tratamentos foram aplicados aos 84 dias após a emergência. As amostragens foram feitas através do método de batida de pano, com cinco batidas ao acaso, por parcela, nas quais foram identificados e contados os predadores vivos. Os inseticidas Clotianidin $500 \mathrm{WP}$ (200 $\left.\mathrm{g} \mathrm{ha}^{-1}\right)$, Carbosulfan $400 \mathrm{SC}$ (400 $\left.\mathrm{mL} \mathrm{ha}^{-1}\right)$, Benfuracarb 400 EC (450 $\left.\mathrm{mL} \mathrm{ha}^{-1}\right)$, Cartap hydrochloride $500 \mathrm{SP}\left(1.000 \mathrm{~g} \mathrm{ha}^{-1}\right)$, Thiamethoxam $250 \mathrm{WG}\left(200 \mathrm{~g} \mathrm{ha}^{-1}\right)$ e Acetamiprid $200 \mathrm{SP}$ $\left(150 \mathrm{~g} \mathrm{ha}^{-1}\right)$ não foram seletivos ao complexo de predadores ocorrentes, com percentagens de mortalidades que oscilaram entre moderadamente tóxicos e tóxicos. Etofenprox $300 \mathrm{EC}$ $\left(450 \mathrm{~mL} \mathrm{ha}^{-1}\right)$ apresentou a maior percentagem de mortalidade, em relação aos demais tratamentos inseticidas, sendo classificado como tóxico. O tratamento Flonicamid $500 \mathrm{WG}$ (150 $\left.\mathrm{g} \mathrm{ha}^{-1}\right)$ foi seletivo, com média de predadores semelhante à testemunha, até 8 dias após a aplicação (daa). Dentre os inseticidas testados, somente o Flonicamid $500 \mathrm{WG}$ foi considerado seletivo, segundo a classificação do IOBC, pois apresentou a menor percentagem de mortalidade ao complexo de predadores ocorrentes.

PALAVRAS-CHAVE: Gossypium hirsutum L.; Insecta; inseticidas; algodão; predadores.

algodoeiro. Para o desenvolvimento sustentado, neste agroecossistema, há necessidade de implementação do Manejo Integrado das Pragas (MIP), que proporciona soluções dos problemas com pragas, a longo prazo. O MIP consiste na integração de práticas e métodos apropriados de controle de pragas, de modo compatível, que possibilita a manutenção das populações dos competidores abaixo do nível de dano econômico. Inclui a com-

1. Trabalho recebido em ago./2009 e aceito para publicação em set./2010 (nº registro: PAT 6860/ DOI: 10.5216/pat.v40i3.6860).

2. Universidade Federal da Grande Dourados, Faculdade de Ciências Agrárias, Setor de Entomologia, Dourados, MS, Brasil.

E-mails: izidrojunior@gmail.com,rfnfernandes@hotmail.com, thiagobertoncello@gmail.com, epmeloagro@yahoo.com.br, rsuekane@hotmail.com, paulodegrande@ufgd.edu.br. 
binação de várias estratégias e táticas de controle, tais como práticas culturais, resistência varietal, controle biológico e manejo de inseticidas (Santos 2001).

É frequente observar-se, na natureza, o controle biológico natural, exercido por inimigos naturais, com potencial para manter, em níveis razoavelmente baixos, as populações de inúmeras pragas. Os inimigos naturais minimizam a necessidade de intervenção do homem, no controle de pragas. Atitudes que visem a manter os inimigos naturais, parasitóides e patógenos de pragas nos agroecossistemas são de fundamental importância para o equilíbrio dinâmico das populações de espécies de insetos e ácaros-praga (Degrande et al. 2002).

Aplicações de produtos fitossanitários de alta toxicidade e largo espectro de ação estão sendo reconhecidas, por diversos autores, como uma das principais causas de desequilíbrios biológicos nos agroecossistemas, provocando fenômenos como ressurgência, aumento de pragas (que normalmente são secundárias) e seleção de insetos resistentes. De acordo com Crocomo (1984), o uso de inseticidas seletivos é de grande importância para retardar, ou mesmo evitar, estes problemas decorrentes do uso de defensivos agrícolas.

A seletividade pode ser definida como a propriedade que um produto fitossanitário apresenta para controlar uma praga específica, com o menor impacto possível sobre os componentes do agroecossistema, ou, ainda, dentro do MIP, a propriedade de possuir baixo efeito sobre os inimigos naturais, nas mesmas condições em que a praga é controlada com sucesso (Gazzoni 1994).

Estudos de seletividade de pesticidas aplicados aos principais inimigos naturais devem ser realizados para o sucesso do MIP, em culturas agrícolas, pois as informações obtidas poderão ser utilizadas nas tomadas de decisão, com relação ao produto a ser aplicado. Estes estudos minimizam os efeitos indesejáveis, decorrentes do uso de pesticidas não apropriados no controle de pragas, uma vez que a seletividade apresenta objetivos conservacionistas dos sistemas de produção, atuando na proteção e no fomento de organismos benéficos (Degrande et al. 2002).

Assim, o objetivo deste trabalho foi estudar a seletividade de alguns inseticidas, com relação aos predadores de pragas do algodoeiro, em condições de campo.

\section{MATERIAL E MÉTODOS}

O experimento foi conduzido a campo, no município de Dourados ( $22^{\circ} 10^{\prime} \mathrm{S}$ e $54^{\circ} 54^{\prime} \mathrm{W}$ ), Mato Grosso do Sul. Foi realizado o preparo convencional do solo, em área de 0,8 ha, aonde foi semeado o algodão, cultivar Delta Opal, em 31/01/2006, com densidade de semeadura de 12 sementes por metro e espaçamento de $0,90 \mathrm{~m}$ entre linhas. $O$ controle de plantas daninhas foi realizado através da aplicação de herbicidas pré e pós-emergente. Anteriormente ao período experimental, não houve controle químico das pragas e doenças foliares ocorrentes, pois o objetivo era estabelecer uma população de inimigos naturais na área. Somente houve intervenção para controle da lagarta curuquerê Alabama argillacea (Hübner) (Lepidoptera: Noctuidae), evitando-se a desfolha acentuada da área experimental, através de pulverizações do inseticida microbiológico Thuricide $^{\circledR}$ (Bacillus thuringiensis - BT), na dosagem de $500 \mathrm{~g} \mathrm{ha}^{-1}$, considerado seletivo aos inimigos naturais (Hassan et al. 1983, Hassan et al. 1994). Os tratamentos utilizados encontram-se descritos na Tabela 1.

O delineamento experimental utilizado foi o de blocos ao acaso, com nove tratamentos e quatro repetições. Os tratamentos foram aplicados aos 84 dias após a emergência, com o uso de equipamento de pressão constante, com propulsão a base de $\mathrm{CO}_{2}$ e bicos do tipo cone vazio JA preto, obedecendo à diluição de $112 \mathrm{~L} \mathrm{ha}^{-1}$, quando foi observado um surto populacional de pulgão (coincidindo com o surto de predadores na área).

As avaliações realizadas foram: prévia, antes da aplicação, e aos 2, 3, 5, 8, 11, 15, 19, 26 e 33 dias após a aplicação (daa). As amostragens foram feitas através do método de batida de pano, com cinco batidas ao acaso por parcela, sendo identificadas e contadas as espécies predadoras vivas.

Para a obtenção da percentagem de mortalidade dos predadores no estudo, foi utilizada a fórmula de Henderson \& Tilton (1955).

Os dados foram submetidos a análise de variância (teste $\mathrm{F}$ ) e as médias, para cada tratamento, comparadas entre si, pelo teste Tukey, ao nível de $5 \%$ de significância.

A classificação dos inseticidas testados, quanto à toxicidade, foi realizada segundo a escala do IOBC, de acordo com Boller et al. (2005), para ensaios de campo (Tabela 2). 
Tabela 1. Produto comercial estudado, com o respectivo ingrediente ativo (I.A.); grupo químico de cada produto e local primário de ação; e dose testada em cada tratamento ( $\mathrm{mL}$ ou g do produto comercial por hectare).

\begin{tabular}{|c|c|c|c|c|c|}
\hline Tratamento & $\begin{array}{l}\text { Produto } \\
\text { comercial }\end{array}$ & I.A. & Grupo químico & Local primário de ação & $\left(\mathrm{mL}\right.$ ou $\left.\mathrm{g} \mathrm{ha} \mathrm{h}^{-1}\right)$ \\
\hline 1 & Safety ${ }^{\circledR} 300 \mathrm{CE}$ & Etofenprox & Piretróides & Moduladores de canais de sódio & 450 \\
\hline 2 & Mospilan $^{\circledR} 200$ SP & Acetamiprid & Neonicotinóides & $\begin{array}{l}\text { Agonistas de receptores nicotínicos } \\
\text { de acetilcolina }\end{array}$ & 150 \\
\hline 3 & Afitrix $^{\circledR} 400 \mathrm{EC}$ & Benfuracarb & Carbamatos & $\begin{array}{l}\text { Inibidores da enzima } \\
\text { acetilcolinesterase }\end{array}$ & 450 \\
\hline 4 & $\operatorname{Cartap}^{\circledR} 500 \mathrm{SP}$ & Cartap hydrochloride & $\begin{array}{l}\text { Análogos de } \\
\text { nereistoxina }\end{array}$ & $\begin{array}{l}\text { Antagonistas de receptores } \\
\text { nicotínicos de acetilcolina }\end{array}$ & 1000 \\
\hline 5 & Marshal $^{\circledR} 400 \mathrm{SC}$ & Carbosulfan & Carbamatos & $\begin{array}{l}\text { Inibidores da enzima } \\
\text { acetilcolinesterase }\end{array}$ & 400 \\
\hline 6 & Actara $^{\circledR} 250 \mathrm{WG}$ & Thiamethoxam & Neonicotinóides & $\begin{array}{l}\text { Agonistas de receptores nicotínicos } \\
\text { de acetilcolina }\end{array}$ & 200 \\
\hline 7 & Turbine $^{\circledR} 500 \mathrm{WG}$ & Flonicamid & Flonicamid & Inibidor seletivo de alimentação & 150 \\
\hline 8 & Focus $^{\circledR} 500 \mathrm{WP}$ & Clothianidin & Neonicotinóides & $\begin{array}{l}\text { Agonistas de receptores nicotínicos } \\
\text { de acetilcolina }\end{array}$ & 200 \\
\hline 9 & Testemunha & - & - & - & - \\
\hline
\end{tabular}

Tabela 2. Classificação da seletividade de pesticidas a inimigos naturais do IOBC, para estudos de campo.

\begin{tabular}{lcc}
\hline \multicolumn{1}{c}{ Classificação } & $\begin{array}{c}\text { Redução na população de } \\
\text { inimigos naturais (\%) }\end{array}$ & Classes \\
\hline Inócuo ou levemente tóxico & $0-50 \%$ & $\mathrm{~N}$ \\
Moderadamente tóxico & $51-75 \%$ & $\mathrm{M}$ \\
Tóxico & $>75 \%$ & $\mathrm{~T}$ \\
\hline
\end{tabular}

Fonte: Boller et al. (2005).

\section{RESULTADOS E DISCUSSÃO}

\section{Complexo de predadores}

Os principais inimigos naturais observados no experimento foram: Cycloneda sanguinea (LINNAEUS, 1763) (Coleoptera: Coccinellidae); Hyppodamia convergens Guérin-Méneville, 1842 (Coleoptera: Coccinellidae); Eriopis conexa (German, 1824) (Coleoptera: Coccinellidae); Scymnus spp. (Coleoptera: Coccinellidae); Geocoris spp. (Hemiptera: Geocoridae); Zellus spp. (Hemiptera: Reduviidae); Podisus spp. (Hemiptera: Pentatomidae); Doru sp. (Dermaptera: Forficulidae); e outros insetos pertencentes às famílias Chrysopidae (Neuroptera) e Syrphidae (Diptera), bem como aranhas (Araneae), corroborando as observações de Nunes et al. (1999) e Czepak et al. (2005), que, também, relataram a presença destes predadores no algodoeiro. Com base nos dados obtidos no experimento, insetos da família Coccinellidae foram os mais abundantes, durante o período de avaliações, atingindo $78 \%$ do total de predadores ocorrentes (Figura 1). Resultados semelhantes foram obtidos por Barros et al. (2006), estudando a flutuação populacional de insetos predadores associados a pragas do algodoeiro, em dois anos agrícolas, nos quais observaram que a população de coccinelídeos também foi a mais abundante, com $79,8 \%$ e $78 \%$ do total de espécimes, em 2001/2002 e 2002/2003, respectivamente.

Para a realização da análise estatística, foi levado em consideração o complexo de inimigos naturais, avaliado de maneira global, somando-se todos os espécimes ocorrentes. Posteriormente, devido ao fato de os dados serem sumarizados em nível de família, fazendo com que os efeitos genuínos possam, muitas vezes, ser negligenciados, foram realizadas análises específicas, para o grupo de predadores predominantes: os coccinelídeos.

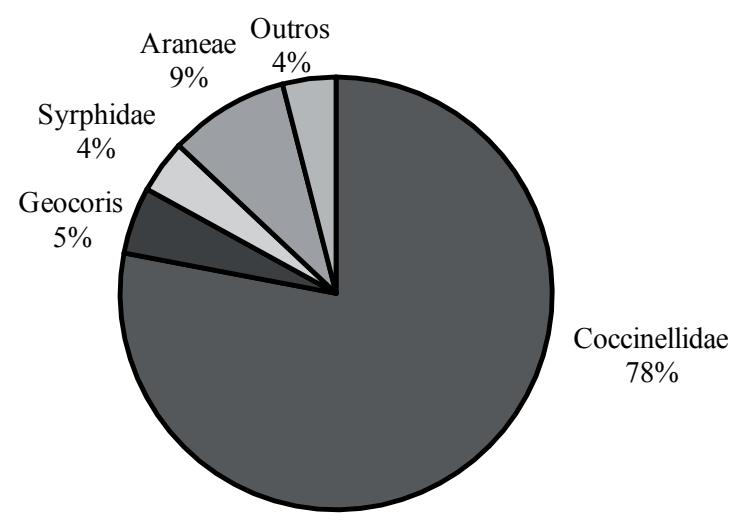

Figura 1. Abundância de predadores das pragas do algodoeiro, durante as avaliações (Dourados, MS, 2006). 
Na avaliação prévia, os valores para o número de predadores, nas parcelas dos diferentes tratamentos, não diferiram, estatisticamente, entre si, demonstrando a uniformidade da população destes predadores, na área experimental (Tabela 3).

Apesar de a coleta de dados ter sido realizada até os 33 daa, as inferências sobre a percentagem de mortalidade foram feitas somente até os 11 daa, pois ocorreu recolonização das parcelas experimentais, impedindo inferências seguras. Esta recolonização também foi relatada por Fonseca et al. (2005) e Lopes (2005), em estudos com a cultura do algodão. A recolonização das parcelas experimentais também é reportada em estudos de seletividade, na cultura da soja (Corso et al. 1999, Batista Filho et al. 2003).

Na Tabela 3, referente ao complexo de predadores, observa-se que, aos 2 daa, o tratamento Etofenprox $300 \mathrm{EC}$ apresentou maior percentagem de mortalidade, alcançando índice de $81 \%$, seguido pelo Carbosulfan 400 SC, com 78\%, sendo ambos classificados como tóxicos (classe T). A baixa seletividade destes produtos, aos 2 daa, pode estar relacionada ao mecanismo e amplo espectro de ação destes piretróides e carbamatos (Gallo et al. 2002). Carvalho et al. (2003) e Pincanço et al. (2003), estudando alguns piretróides e carbamatos, respectivamente, também observaram a não seletividade de alguns inseticidas destes grupos.

Os tratamentos Benfuracarb 400 EC, Clothianidin 500 WP, Cartap hydrochloride 500
SP e Thiamethoxam $250 \mathrm{WG}$ foram classificados como moderadamente tóxicos (classe M). Os produtos Acetamiprid 200 SP e Flonicamid 500 WG foram, segundo a tabela de classificação, inócuos ou levemente tóxicos (classe $\mathrm{N}$ ) ao complexo de predadores, destacando-se a baixa mortalidade atingida pelo Flonicamid $500 \mathrm{WG}$, que foi de apenas $4 \%$, não diferindo, estatisticamente, da testemunha (Tabela 3).

De acordo com Staetz et al. (2006), o Flonicamid é um produto sistêmico, que bloqueia os canais de potássio Tipo-A de homópteros e hemípteros, o que altera a transmissão sináptica normal e conduz à perda do controle do sistema nervoso, fazendo com que o inseto cesse a alimentação, previamente ao dano, seguido de morte. Ainda, segundo os mesmos autores, este produto possui um método único de ação, o que o torna seletivo a outros insetos não alvos.

Os resultados observados aos 3 daa foram semelhantes aos descritos anteriormente, exceto para o Carbosulfan $400 \mathrm{SC}$, que passou da classe T para M. Observou-se, novamente, o reduzido impacto dos produtos Acetamiprid $200 \mathrm{SP}$ e Flonicamid 500 WG, que apresentaram os menores índices de mortalidade. Torres et al. (2002), estudando toxicidade de inseticidas do mesmo grupo que o Flonicamid, ao percevejo Podisus spp., observaram que, aos 3 daa, houve sobrevivência do predador acima de $80 \%$. Etofenprox 300 EC continuou muito tóxico aos predadores (classe T) (Tabela 3).

Tabela 3. Número médio de predadores por parcela (x) e percentagem de mortalidade $(\% \mathrm{M})$ do complexo de predadores ocorrentes na avaliação prévia e 2, 3, 5, 8 e 11 dias após a aplicação, nos tratamentos testados (Dourados, MS, 2006).

\begin{tabular}{|c|c|c|c|c|c|c|c|c|c|c|c|c|}
\hline \multirow{3}{*}{ Tratamento } & \multirow{3}{*}{ Dose } & \multirow{3}{*}{$\begin{array}{c}\begin{array}{c}\text { Avaliação } \\
\text { Prévia }\end{array} \\
\mathrm{X}^{1}\end{array}$} & \multicolumn{10}{|c|}{ Dias após a aplicação (daa) } \\
\hline & & & \multicolumn{2}{|c|}{2 daa } & \multicolumn{2}{|c|}{3 daa } & \multicolumn{2}{|c|}{5 daa } & \multicolumn{2}{|c|}{8 daa } & \multicolumn{2}{|c|}{11 daa } \\
\hline & & & $\mathrm{x}$ & $\% \mathrm{M}$ & $\mathrm{X}$ & $\% \mathrm{M}$ & $\mathrm{x}$ & $\% \mathrm{M}$ & $\mathrm{X}$ & $\% \mathrm{M}$ & $\mathrm{X}$ & $\% \mathrm{M}$ \\
\hline Etofenprox $300 \mathrm{EC}$ & 450 & $48,5 \mathrm{a}^{2}$ & $10,5 \mathrm{~b}$ & 81 & $7,3 \mathrm{~d}$ & 86 & $8,3 \mathrm{~d}$ & 84 & $9,0 \mathrm{~d}$ & 83 & $7,3 \mathrm{~b}$ & 81 \\
\hline Acetamiprid $200 \mathrm{SP}$ & 150 & $40,5 \mathrm{a}$ & $26,5 \mathrm{ab}$ & 41 & $30,8 \mathrm{abc}$ & 27 & $21,0 \mathrm{bcd}$ & 52 & $17,5 \mathrm{bcd}$ & 61 & $11,3 \mathrm{~b}$ & 65 \\
\hline Benfuracarb $400 \mathrm{EC}$ & 450 & $45,0 \mathrm{a}$ & $13,3 \mathrm{~b}$ & 74 & 19,0 abcd & 60 & $21,8 \mathrm{~cd}$ & 56 & $18,8 \mathrm{bcd}$ & 62 & $12,0 \mathrm{~b}$ & 66 \\
\hline Cartap hydrochloride 500 SP & 1000 & $57,8 \mathrm{a}$ & $20,8 \mathrm{ab}$ & 68 & 17,8 abcd & 71 & $26,0 \mathrm{abc}$ & 59 & $23,3 \mathrm{bc}$ & 63 & $19,3 \mathrm{ab}$ & 58 \\
\hline Carbosulfan $400 \mathrm{SC}$ & 400 & $45,0 \mathrm{a}$ & $11,3 \mathrm{~b}$ & 78 & $15,5 \mathrm{bcd}$ & 67 & $16,8 \mathrm{~cd}$ & 66 & $13,8 \mathrm{bcd}$ & 72 & $12,0 \mathrm{~b}$ & 66 \\
\hline Thiamethoxam $250 \mathrm{WG}$ & 200 & $54,8 \mathrm{a}$ & $27,5 \mathrm{ab}$ & 55 & 28,0 abcd & 51 & $23,8 \mathrm{abcd}$ & 60 & $22,5 \mathrm{bc}$ & 62 & $12,3 \mathrm{~b}$ & 72 \\
\hline Flonicamid $500 \mathrm{WG}$ & 150 & $43,0 \mathrm{a}$ & $46,0 \mathrm{a}$ & 4 & $41,8 \mathrm{ab}$ & 7 & $45,5 \mathrm{ab}$ & 3 & $28,0 \mathrm{ab}$ & 40 & $11,0 \mathrm{~b}$ & 68 \\
\hline Clothianidin $500 \mathrm{WP}$ & 200 & $45,5 \mathrm{a}$ & $12,5 \mathrm{~b}$ & 75 & $14,3 \mathrm{~cd}$ & 70 & $12,3 \mathrm{~cd}$ & 75 & $11,0 \mathrm{~cd}$ & 78 & $8,5 \mathrm{~b}$ & 76 \\
\hline Testemunha & - & $42,5 \mathrm{a}$ & $47,3 \mathrm{a}$ & - & $44,5 \mathrm{a}$ & - & $46,3 \mathrm{a}$ & - & $46,5 \mathrm{a}$ & - & $33,5 \mathrm{a}$ & - \\
\hline Valor de F & - & 0,57 ns & $7,05 * *$ & - & $6,18^{* *}$ & - & $8,70 * *$ & - & $10,71 * *$ & - & $7,37 * *$ & - \\
\hline C.V. $(\%)$ & - & 16,05 & 22,24 & - & 21,66 & - & 18,10 & - & 15,65 & - & 18,62 & - \\
\hline
\end{tabular}

Os dados originais das médias foram transformados em raiz quadrada de $(\mathrm{x}+0,5)$, para fins de análise estatística.

${ }^{2}$ Médias seguidas de mesma letra, na coluna, não diferem entre si, pelo teste Tukey, a $5 \%$ de probabilidade.

ns: Não significativo ao teste F, a $5 \%$ de probabilidade; **: Significativo ao teste F, a $1 \%$ de probabilidade. 
Aos 5 daa, o tratamento Etofenprox 300 EC continuou apresentando a maior percentagem de mortalidade (classe T). O tratamento Flonicamid 500 WG foi novamente inócuo (classe N), com média de predadores semelhante à da testemunha. Os demais tratamentos foram classificados como medianamente tóxicos (classe M).

$\mathrm{Na}$ avaliação aos 8 daa, foi observado o início de uma diferença entre os tratamentos, devido à eficácia dos produtos, pois alguns produtos não foram efetivos no controle do pulgão, o que permitiu grande oferta de alimento aos predadores, nestas parcelas. Em contrapartida, em outras parcelas, alguns produtos efetivos para o controle desta praga diminuíram a oferta de alimento, provocando migração de inimigos naturais das parcelas, devido à falta de alimento, o que pode ter interferido na interpretação dos resultados.

De acordo com Degrande (1993), a presença de predadores está relacionada com a disponibilidade de alimento, no caso, as pragas. O autor constatou que Coccinellidae, Chrysopidae e Syrphidae estavam associados às populações de $A$. gossypii, enquanto Geocoris spp. estava associado à presença das lagartas de $H$. virescens e $A$. argillacea, demonstrando que o grupo de predadores predominante em determinada fase de desenvolvimento da cultura está relacionado com a espécie de praga predominante nesta fase. Isto também foi observado por Barros et al. 2006.

Os resultados com Thiamethoxam $250 \mathrm{WG}$ diferiram dos encontrados por Czepak et al. (2005), onde thiamethoxan foi considerado seletivo ao complexo de inimigos naturais, com $0 \%$ de mortalidade, aos 3 daa, e $25,1 \%$, aos 8 daa, o que o colocaria na classe $\mathrm{N}$, nas duas avaliações, segundo a classificação utilizada neste trabalho (Tabela 3 ).

As avaliações de 8 a 11 daa apresentaram resultados semelhantes. O produto Clothianidin 500 WP passou da classe M para a classe T, juntamente com o Etofenprox 300 EC. Os demais tratamentos se mantiveram na classe $\mathrm{M}$, nas duas avaliações, exceto o Turbine $500 \mathrm{WG}$, que passou da classe N, na avaliação aos 8 daa, para a classe $\mathrm{M}$, aos 11 daa. Esta migração de classe do Flonicamid $500 \mathrm{WG}$ pode estar relacionada ao efeito do produto no controle da praga, pois o produto eliminou a praga, fazendo com que limitasse a oferta de alimento aos predadores, como já descrito anteriormente.

A comparação de resultados, nos estudos de seletividade, em alguns casos, torna-se pouco conveniente, devido à pluralidade de produtos, insetos e, principalmente, das metodologias empregadas nestes estudos. Esse fato fica evidenciado observando-se importantes pesquisas como as de Carvalho et al. (2003) e Ulhôa et al. (2002). Carvalho et al. (2003), estudando o efeito de esfenvalerate sobre ninfas de Chrysopidae, até terceiro instar, observaram a seletividade deste produto. No entanto, Ulhôa et al. (2002), trabalhando com o mesmo produto, dose, metodologia e mesmo inimigo natural, porém, em diferente estágio de desenvolvimento, reportaram efeito altamente tóxico do inseticida.

Tabela 4. Número médio de Coccinellidae por parcela $(\mathrm{x})$ e percentagem de mortalidade $(\% \mathrm{M})$ do complexo de inimigos naturais, ocorrentes na avaliação prévia e 2, 3, 5, 8 e 11 dias após a aplicação, nos tratamentos testados (Dourados, MS, 2006).

\begin{tabular}{|c|c|c|c|c|c|c|c|c|c|c|c|c|}
\hline \multirow{3}{*}{ Tratamento } & \multirow{3}{*}{ Dose } & \multirow{3}{*}{$\begin{array}{c}\text { Avaliação } \\
\text { prévia }\end{array}$} & \multicolumn{10}{|c|}{ Dias após a aplicação } \\
\hline & & & \multicolumn{2}{|l|}{2} & \multicolumn{2}{|l|}{3} & \multicolumn{2}{|l|}{5} & \multicolumn{2}{|l|}{8} & \multicolumn{2}{|l|}{11} \\
\hline & & & $\mathrm{x}$ & $\% \mathrm{M}$ & $\mathrm{x}$ & $\% \mathrm{M}$ & $\mathrm{x}$ & $\% \mathrm{M}$ & $\mathrm{x}$ & $\% \mathrm{M}$ & $\mathrm{x}$ & $\% \mathrm{M}$ \\
\hline Etofenprox $300 \mathrm{EC}$ & 450 & $41,5 \mathrm{a}^{2}$ & $6,0 \mathrm{~b}$ & 87 & $3,3 \mathrm{c}$ & 93 & $3,5 \mathrm{c}$ & 92 & $6,0 \mathrm{~d}$ & 87 & $2,3 \mathrm{c}$ & 93 \\
\hline Acetamiprid $200 \mathrm{SP}$ & 150 & $32,3 \mathrm{a}$ & $19,8 \mathrm{ab}$ & 47 & $21,8 \mathrm{abc}$ & 39 & $16,5 \mathrm{abc}$ & 53 & $11,8 \mathrm{bcd}$ & 68 & $4,3 \mathrm{bc}$ & 83 \\
\hline Benfuracarb $400 \mathrm{EC}$ & 450 & $34,3 \mathrm{a}$ & $8,3 \mathrm{~b}$ & 79 & $11,5 \mathrm{abc}$ & 70 & $15,3 \mathrm{abc}$ & 59 & $13,0 \mathrm{bcd}$ & 66 & $7,0 \mathrm{bc}$ & 73 \\
\hline Cartap hydrochloride $500 \mathrm{SP}$ & 1000 & $48,5 \mathrm{a}$ & $17,3 \mathrm{ab}$ & 69 & $13,8 \mathrm{abc}$ & 74 & $18,8 \mathrm{ab}$ & 65 & $19,0 \mathrm{abc}$ & 65 & $14,8 \mathrm{ab}$ & 60 \\
\hline Carbosulfan $400 \mathrm{SC}$ & 400 & $38,0 \mathrm{a}$ & $8,3 \mathrm{~b}$ & 81 & $10,0 \mathrm{bc}$ & 76 & $10,0 \mathrm{bc}$ & 76 & $7,8 \mathrm{bcd}$ & 82 & $5,5 \mathrm{bc}$ & 81 \\
\hline Thiamethoxam $250 \mathrm{WG}$ & 200 & $44,5 \mathrm{a}$ & $22,3 \mathrm{ab}$ & 57 & $24,8 \mathrm{ab}$ & 50 & $19,3 \mathrm{ab}$ & 60 & $17,3 \mathrm{bcd}$ & 65 & $11,3 \mathrm{abc}$ & 67 \\
\hline Flonicamid $500 \mathrm{WG}$ & 150 & $34,5 \mathrm{a}$ & $41,8 \mathrm{a}$ & 0 & $37,0 \mathrm{a}$ & 3 & $37,3 \mathrm{a}$ & 1 & $22,0 \mathrm{ab}$ & 43 & $5,8 \mathrm{bc}$ & 78 \\
\hline Clothianidin $500 \mathrm{WP}$ & 200 & $36,0 \mathrm{a}$ & $9,3 \mathrm{~b}$ & 78 & $10,8 \mathrm{abc}$ & 73 & $8,3 \mathrm{bc}$ & 79 & $7,3 \mathrm{~cd}$ & 82 & $5,0 \mathrm{bc}$ & 82 \\
\hline Testemunha & - & $33,0 \mathrm{a}$ & $38,0 \mathrm{a}$ & & $36,5 \mathrm{a}$ & & $36,0 \mathrm{a}$ & & $37,0 \mathrm{a}$ & & $25,3 \mathrm{a}$ & \\
\hline $\mathrm{F}$ & - & $0,64^{\mathrm{ns}}$ & $7,51 * *$ & & $5,43 * *$ & & $7,99 * *$ & & $9,01 * *$ & & $7,89 * *$ & \\
\hline C.V. $(\%)$ & - & 17,91 & 24,90 & & 29,24 & & 23,52 & & 20,27 & & 27,08 & \\
\hline
\end{tabular}

${ }^{1}$ Os dados originais das médias foram transformados em raiz quadrada de $\mathrm{x}+0,5$, para fins de análise estatística.

${ }^{2}$ Médias seguidas de mesma letra, na coluna, não diferem entre si, pelo teste Tukey, a $5 \%$ de probabilidade.

ns: Não significativo ao teste F, a $5 \%$ de probabilidade; **: Significativo ao teste F, a $1 \%$ de probabilidade. 


\section{Coccinellidae}

A população inicial de Coccinellidae na área apresentou-se uniforme, na avaliação prévia, não havendo diferença estatística entre os tratamentos (Tabela 4). O número médio de espécimes variou entre 32,3 e 48,5. Na avaliação aos 2 daa, os tratamentos Etofenprox 300 EC, Carbosulfan 400 SC, Benfuracarb 400 EC e Clothianidin 500 WP, com $87 \%, 81 \%, 79 \%$ e $78 \%$ de mortalidade, foram os mais impactantes à Coccinellidae, classe $\mathrm{T}$, com médias estatisticamente semelhantes entre si e diferentes, em relação à testemunha. Já os produtos Flonicamid 500 WG e Acetamiprid 200 SP, alocados na classe N, com $0 \%$ e $47 \%$ de mortalidade, respectivamente, foram inócuos a este grupo de predadores e apresentaram médias semelhantes à da testemunha. Os demais produtos foram classificados como medianamente tóxicos (classe M).

Os produtos testados apresentaram resultados semelhantes, nas avaliações aos 3 e 5 daa. Os produtos Flonicamid $500 \mathrm{WG}$ e Acetamiprid 200 SP continuaram inócuos, juntamente com o Thiamethoxam $250 \mathrm{WG}$, aos 3 daa. Etofenprox 300 EC e Carbosulfan $400 \mathrm{SC}$ se mantiveram tóxicos. O Cartap hydrochloride 500 SP e Benfuracarb 400 EC, aos 3 e 5 daa, e Thiamethoxam $250 \mathrm{WG}$, aos 5 daa, foram classificados como moderadamente tóxicos. Clothianidin 500 WP oscilou entre M, aos 3 daa, e $\mathrm{T}$, aos 5 daa.

Aos 8 daa, somente o Flonicamid 500 WG foi inócuo às joaninhas, com média semelhante à da testemunha. Já Etofenprox 300 EC, Carbosulfan 400 $\mathrm{SC}$ e Clothianidin $500 \mathrm{WP}$ apresentaram mortalidade de $87 \%, 82 \%$ e $82 \%$, respectivamente. Os demais tratamentos foram alocados na classe $\mathrm{M}$.

$\mathrm{Na}$ avaliação aos 11 daa, os tratamentos Cartap hydrochloride 500 SP, Thiamethoxam 250 WG e Benfuracarb 400 EC foram alocados na classe $\mathrm{M}$, enquanto os demais produtos foram classificados como $\mathrm{T}$, inclusive Flonicamid $500 \mathrm{WG}$ e Acetamiprid 200 SP. A inclusão de Flonicamid 500 WG e Acetamiprid 200 SP pode estar relacionada à eficiência dos produtos, pois, com a eliminação da praga, os inimigos naturais estariam migrando das parcelas que receberam estes tratamentos.

Observou-se que, no estudo isolado de Coccinellidae, o Carbosulfan 400 SC foi classificado como tóxico, aos 11 daa, o que não ocorreu quando se analisou o complexo de predadores, evidenciando que este produto é mais impactante para Coccinellidae. Este fato pode estar relacionado à formulação do produto, pois, segundo Stainier et al. (2006), estudando o efeito de diferentes formulações nas pulverizações, o tamanho e a deposição de gotas são alterados.

\section{CONCLUSÕES}

1. O inseticida Flonicamid $500 \mathrm{WG}$ é seletivo e Etofenprox 300 EC tóxico ao complexo de predadores encontrado na cultura do algodoeiro, em Dourados, MS.

2. Os demais produtos (Clothianidin $500 \mathrm{WP}$, Carbosulfan 400 SC, Benfuracarb 400 EC, Cartap hydrochloride $500 \mathrm{SP}$, Thiamethoxam $250 \mathrm{WG}$ e Acetamiprid $200 \mathrm{SP}$ ) são moderadamente tóxicos.

\section{REFERÊNCIAS}

BARROS, R. et al. Flutuação populacional de insetos predadores associados às pragas do algodoeiro. Arquivos do Instituto Biológico, São Paulo, v. 73, n. 1, p. 57-64, 2006.

BATISTA FILHO, A. et al. Manejo integrado de pragas em soja: impacto de inseticidas sobre inimigos naturais. Arquivos do Instituto Biológico, São Paulo, v. 70, n. 1, p. 61-67, 2003.

BOLLER, E. F. et al. Working document on selectivity of pesticides. 2005. Disponível em: <http://www. iobc.ch/2005/Working\%20Document $\% 20$ Pesticides_ Explanations.pdf $>$. Acesso em: 25 ago 2009.

CARVALHO, G. A. et al. Efeitos de inseticidas usados na cultura do algodoeiro sobre Chrysoperla externa (Hagen) (Neuroptera: Chrysopidae). Neotropical Entomology, Londrina, v. 32, n. 4, p. 699-706, 2003.

CORSO, I. C.; GAZZONI, D. L.; NERY, M. E. Efeito de doses e de refúgio sobre a seletividade de inseticidas a predadores e parasitóides de pragas de soja. Pesquisa Agropecuária Brasileira, Brasília, DF, v. 34, n. 9, p. 15291538, 1999.

CROCOMO, W. B. Manejo de pragas. In: CROCOMO, W. B. (Org.). O que é manejo de pragas. Botucatu: Unesp, 1984. p. 1-16.

CZEPAK, C. et al. Seletividade de inseticidas ao complexo de inimigos naturais na cultura do algodão (Gossypium hirsutum L.). Pesquisa Agropecuária Tropical, Goiânia, v. 35, n. 2, p. 123-127, 2005. 
DEGRANDE, P. E. Validação do manejo integrado de pragas aplicado ao algodoeiro no Mato Grosso do Sul através de campos demonstrativos. In: CONGRESSO BRASILEIRO DE ENTOMOLOGIA, 14., 1993, Piracicaba. Resumos... Piracicaba: SEB, 1993. p. 574.

DEGRANDE, P. E. et al. Metodologia para avaliar o impacto de pesticidas sobre inimigos naturais. In: PARRA, J. R. P. et al. (Eds.). Controle biológico no Brasil: parasitóides e predadores. São Paulo: Manole, 2002. p. 71-94.

FONSECA, P. R. B. et al. Recolonização de unidades experimentais por predadores de pragas após a aplicação de inseticida no algodoeiro visando estudos de seletividade. In: CONGRESSO BRASILEIRO DE ALGODÃO, 5., 2005, Salvador. Resumos... Salvador: Embrapa, 2005. p. 22.

GALLO, D. et al. Entomologia agrícola. Piracicaba: Fealq, 2002.

GAZZONI, D. L. Pesquisa em seletividade de inseticidas no Brasil. In: SIMPÓSIO DE CONTROLE BIOLÓGICO, 4., 1994, Gramado. Anais... Pelotas: Embrapa-CPACT, 1994. p. 119-124.

HASSAN, S. A. et al. Results of the second joint pesticide testing programme by the IOBC/WPRS Working Group "Pesticides and Beneficial Arthropods". Journal of Applied Entomology, Zeitschrift, v. 95, n. 1, p. 151-158, 1983.

HASSAN, S. A. et al. Results of the sixth joint pesticide testing programme carried out by the IOBC/WPRS Working Group "Pesticides and Beneficial Organisms". Entomophaga, Paris, v. 39, n. 1, p. 107-119, 1994.

HENDERSON, C. F.; TILTON, E. W. Tests with acaricides against the brown wheat mite. Journal of Economic Entomology, Lanham, v. 48, n. 2, p. 157-161, 1955.

LOPES, J. Metodologia experimental para avaliação de seletividade de inseticidas em predadores de pragas no algodoeiro. 2005. 71 f. Dissertação (Mestrado em Produção Vegetal)-Universidade Federal do Mato Grosso do Sul, Dourados, 2005.
NUNES, J. C. S. et al. Seletividade de inseticidas aos predadores das pragas do algodoeiro. Pesquisa Agropecuária Tropical, Goiânia, v. 29, n. 1, p. 71-75, 1999.

PICANÇO, M. C. et al. Seletividade de inseticidas a Doru luteips (Scudder, 1876) (Dermaptera: Forficulidae) e Cotesia sp. (Hymenoptera: Braconidae) inimigos naturais de Ascia monuste orseis (Godart, 1818) (Lepdoptera: Pieridae). Ciência Rural, Santa Maria, v. 33, n. 2, p. 183188, 2003.

SANTOS, W. J. Identificação, biologia, amostragem e controle das pragas do algodoeiro. In: EMBRAPA. Algodão: tecnologia de produção. Dourados: Embrapa Agropecuária Oeste, 2001. p. 181-226.

STAETZ, C. et al. Flonicamid: a novel mode of action for piercing sucking insects. In: BELTWIDE COTTON CONFERENCES,1., 2006, San Antonio. Proceedings... Memphis: National Cotton Council, 2006. p. 15831590.

STAINIER C. et al. Droplet size spectra and drift effect of two phenmedipham formulations and four adjuvants mixtures. Crop Protection, Amsterdam, v. 25, n. 12, p. 1238-1243, 2006.

TORRES, J. B. et al. Compatibilidade de inseticidas e acaricidas com o percevejo predador Podisus nigrispinus (Dallas) (Heteroptera: Pentatomidae) em algodoeiro. Neotropical Entomoly, Londrina, v. 31, n. 2, p. 311-317, 2002.

ULHÔA, J. L. R. et al. Ação de inseticidas recomendados para o controle do curuquerê-do-algodoeiro para pupas e adultos de Chrysoperla externa (Hagen, 1861) (neuroptera: chrysopidae). Ciência e Agrotecnologia, Lavras, ed. especial, p. 1365-1372, 2002. 